\title{
Full wave modeling of Doppler backscattering from filaments
}

V. Bulanin ${ }^{1}$, E. Gusakov², V. Gusev ${ }^{2}$, G. Zadvitskiy ${ }^{3,4}$, C. Lechte ${ }^{5}$, S. Heuraux ${ }^{3}$, V. Minaev²,

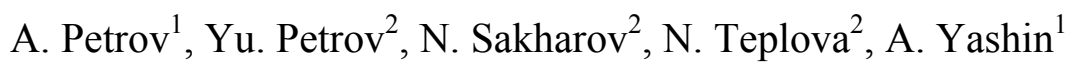

${ }^{I}$ Peter the Great St. Petersburg Polytechnic University, St. Petersburg, Russia

${ }^{2}$ Ioffe Institute, St. Petersburg, Russia

${ }^{3}$ Institut Jean Lamour CNRS-Université de Lorraine, F-54011 Nancy, France ${ }^{5}$

${ }^{4}$ Institute of Plasma Physics of the CAS, Prague, Czech Republic

${ }^{5}$ Institute of Interfacial Process Engineering and Plasma Technology, Stuttgart, Germany

\begin{abstract}
It is recognized that the filaments have a significant effect on the anomalous energy and particle transport in the tokamak periphery. They are actively investigated using various diagnostics in this regard. Recently studies of filaments using the Doppler backscattering (DBS) method have been performed in the Globus-M and the ASDEX-Upgrade tokamaks. Backscattering from filaments manifests itself as a burst of quasi-coherent fluctuations (BQF) of the signals of IQ detectors. Such signals are easy to describe in the Born approximation using the diagnostic weighting function. However, the filaments in tokamaks differ noticeably in their size and intensity. With an increase in the amplitude of the filaments, it is necessary to consider the transition from linear scattering to nonlinear one and further up to the transition from backscattering to reflection from a moving over dens filament. This problem can be solved only using a full wave code. Our simulation was carried out using the finite-difference time-domain code IPF-FD3D in slab geometry. We did not resort to using well-known nonlinear MHD codes to determine filament parameters. In the simulation artificial filament-like perturbations were used, the parameters of which varied over a wide range. Modeling DBS signal was focused on the identification of the influence of the amplitude of the filament and its size on the shape and the size of the DBS output signal. The results obtained largely explain the similarity of the IQ detector data registered in different tokamaks.
\end{abstract}

\section{Introduction}

Filamentary-like plasma perturbations are routinely observed in many tokamaks. They are the result of non-linear development of some peripheral MHD instabilities in the region of the maximum plasma pressure gradient [1]. As filaments can play a key role in the anomalous transport of particles and energy at plasma periphery studies of filaments are actively continuing in various tokamak experiments for better understanding of filament physics and extrapolating of filament parameters to the tokamaks of ITER scale [2]. Various diagnostics are employed for this purpose. A few years ago the authors proposed to use the Doppler 
backscattering (DBS) method for filaments investigation in the Globus-M spherical tokamak $[3,4]$. Later this diagnosis was successfully applied to the study of the filaments in the ASDEX Upgrade tokamak [5, 6]. Backscattering from filaments manifests itself as a burst of quasi-coherent fluctuations (BQF) of the signals of IQ (In-phase and Quadrature) detectors. Such signals are easy to describe in the Born approximation in a case of linear backscattering [3]. Basing on the concept of linear scattering it is possible to determine the position and velocity of the filament. However, the filaments in tokamaks differ noticeably in their size and intensity. With an increase in the amplitude of the filaments, it is necessary to consider the transition from linear scattering to nonlinear one and further up to the transition from backscattering to reflection from a moving filament. This problem can be solved only using a full wave code.

Several studies on full wave modeling of DBS are known [7], [8]. In these works various representations of scattering turbulent plasma fluctuations in toroidal plasma magnetic confinement systems were used. However only one short report in which an attempt to simulate backscattering on filaments of a specific shape is known [6]. In the present paper the IPF-FD3D full wave code [9] was used for modeling of DBS in case of O-mode probing for the purpose of DBS output calculation for various values of the filament density, size and position relatively the cut off of the oblique incident microwave radiation. The geometric parameters of the diagnostic scheme and the radial distribution of the plasma density were chosen close to the parameters of the experiments on the Globus-M spherical tokamak. The paper consists of two parts. In the first part the adaptation of the full-wave code to simulate Doppler backscattering by filaments and the model of a filament are described. The second part presents the simulation results. In the conclusion the degree to which modeling contributes to an adequate interpretation of experimental data is discussed.

\section{Numerical analysis}

The 2D calculation of shape and intensity with the help of non-linear MHD codes was not used to describe the filaments in this work. Such calculations, unfortunately, do not determine the movement of filaments, which is necessary to restore temporary DBS output signals. Instead we have used relatively simple model of a filament with a 2D Gaussian cross-section that allows freedom in choosing the size, amplitude and position of a filament.

Two-dimensional full wave simulations are done with finite-difference time-domain code IPF-FD3D [9] in slab geometry. The calculation of the electric field was carried out in slab geometry in a box of $30 \times 25 \mathrm{~cm}^{2}$. The $\mathrm{x}$ axis corresponds to the poloidal direction in the tokamak, and the y axis corresponds to the radial direction. The parameters of the DBS 
scheme are as following: antenna tilt angle $\alpha=13^{\circ}$, antenna horn mouth $5.5 \mathrm{~cm}$, Gaussian beam with flat wave front in the antenna mouth. The antenna horn mouth is shown by a solid line in figure 1a and $\mathrm{b}$. Computations have been performed for the frequencies $16 \mathrm{GHz}, 24$ $\mathrm{GHz}, 32 \mathrm{GHz}, 40 \mathrm{GHz}, 48 \mathrm{GHz}, 56 \mathrm{GHz}, 64 \mathrm{GHz}$ in O-mode that corresponds to the frequency range used in the Globus-M tokamak DBS diagnostics $(20 \mathrm{GHz}, 29 \mathrm{GHz}, 39 \mathrm{Ghz}$ and $48 \mathrm{GHz}$ ). Experimental plasma density profile used for the computations close to linear, $\mathrm{n}_{\mathrm{e}}=5.6 \cdot 10^{19}$ $\mathrm{cm}^{-3}$ at $\mathrm{r}=0.15 \mathrm{~m}$ (shot\#36569 at Globus-M) is shown in figure 1 by color bar. The filament moves in poloidal direction at $\mathrm{r}=0.092 \mathrm{~m}$, the direction of motion is shown in figure 1 by an arrow. Thus, the radial position of the filament trajectory relative to the cutoff changed with a change in the probe frequency. The imitation of the filament motion was simulated by independent snapshots with a spatial step $1.3 \mathrm{~mm}$. If a time interval assigned to this step the velocity of filament and IQ signals can be determined. For each snapshot the values of IQ signals were calculated (amplitude and phase or I and Q signals) modeling a time dependency $\mathrm{I}(\mathrm{t})$ and $\mathrm{Q}(\mathrm{t})$. Various filament amplitudes $(0.1 \%, 1 \%, 5 \%, 50 \%, 100 \%$ and $150 \%$ of density at cutoff of the probing wave) were set during the simulation in order to reveal the transition from linear to nonlinear scattering.

In Figure 1 the example of superimposition of the electric field of incident, reflected and backscattered waves is shown. The distribution was calculated for filament width $1.5 \mathrm{~cm}$ at probing frequency $48 \mathrm{GHz}$ in the both linear (Figure 1(a)) and non-linear (Figure 1(b)) scattering regime. The relevant filament amplitudes were $0.1 \%$ and $50 \%$ from the density at cut off. The yellow circle shows schematically the position and circular shape of the filament. Field distortion is clearly seen in the nonlinear case.

\section{Results of simulations}

Full-wave modeling was originally undertaken for linear scattering. In the linear case, the manifestation of DBS in the form of BQF can be explained on the basis of the concept of the weighting function [7, 10]. For example, the distribution shown in Figure 1a corresponds to the $2 \mathrm{D}$ alternating weighting function. Since the IQ signals in the Born approximation are represented as the integral of the product of the plasma perturbation and the weighting function [10], the filament motion along the cutoff (see Figure 1a) leads to the appearance of BQF (see Figure 2a and b, blue). At a small filament amplitude, scattering occurs mainly in the cutoff region, and the Doppler shift is related to the filament velocity by the relation $\Delta \omega_{\mathrm{D}}$ $=\mathbf{k}_{\perp} \mathbf{V}_{\perp}, \mathbf{k}_{\perp}=2 \mathbf{k}_{\mathrm{i}}$. Here $\mathbf{k}_{\mathrm{i}}$ is the wave vector of the incident radiation in the cutoff, and $\mathrm{k}_{\perp}=\mathrm{k}_{\mathrm{x}}$ $=2 \mathrm{k}_{0} \sin \alpha$ ( $\mathrm{k}_{0}$ is a free space wavelength of the incident radiation) in case of slab geometry. 

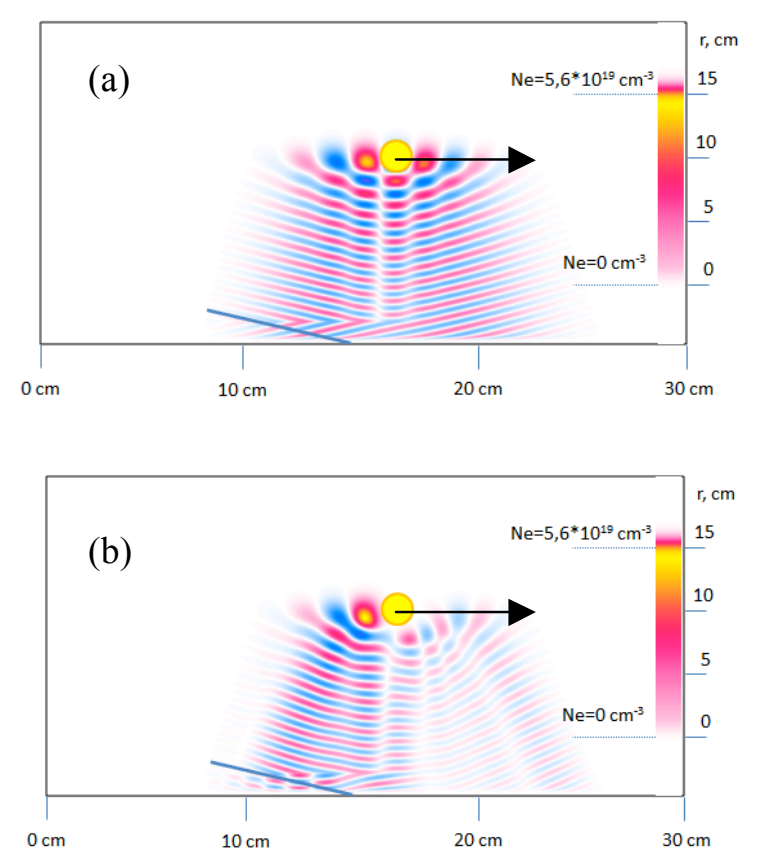

Figure 1. Electric field distribution for linear (a) and non-linear (b) regime.
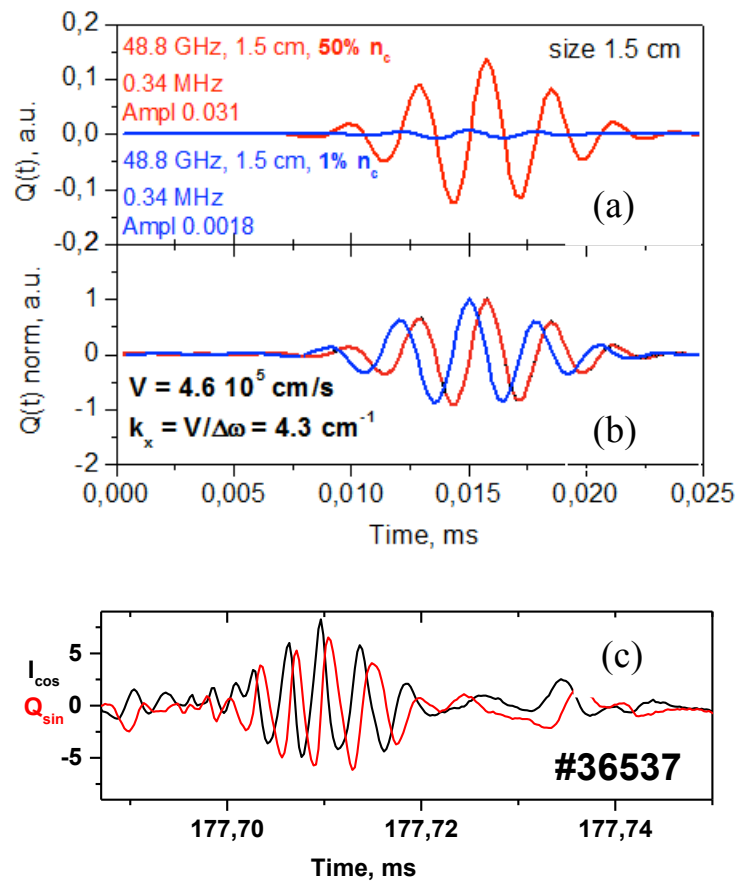

Figure 2. (a) Calculated $\mathrm{Q}(\mathrm{t})$ signals for linear (blue) and nonlinear(red) regimes; (b) normalized calculated signals; (c) $\mathrm{I}(\mathrm{t})$ and $\mathrm{Q}(\mathrm{t})$ DBS signals measured in Globus-M shot\#36537, f=48GHz.

Calculations carried out using the full-wave code for the $1.5 \mathrm{~cm}$ circular filament with a filament amplitude of $1 \%$ have demonstrated the appearance of BQF (see Figure 2a). The size of $1.5 \mathrm{~cm}$ was close to the spatial half-period of the weight function in the cutoff $-\pi / 2 \mathrm{k}_{\mathrm{i}} \sin \alpha$. The calculations were performed for a filament moving at the cutoff radius for $48 \mathrm{GHz}$ radiation, i.e. at $\mathrm{y}=\mathrm{r}=9 \mathrm{~cm}$ wherein the relation $\Delta \omega_{\mathrm{D}}=\mathbf{k}_{\perp} \mathbf{V}_{\perp}$ is fulfilled with great accuracy. The use of full-wave modeling allows one to answer the question of whether the BQF distortions occur and to what extent the relation $\Delta \omega_{D}=\mathbf{k}_{\perp} \mathbf{V}_{\perp}$ is fulfilled with increasing filament amplitude, i.e. upon the transition to the nonlinear scattering regime. Calculations performed for a filament of the same size $1.5 \mathrm{~cm}$ and moving along the same trajectory have shown that BQF are also observed at an amplitude of 50\% (see Figure 2a). In this case, a significant distortion of the superposition of electric fields was observed (see Figure 1b), and the amplitude of quasi-coherent oscillations increased significantly, as can be seen in Figure $2 \mathrm{a}$.

After normalization BQFs for the both linear and nonlinear look similar as it is shown in figure 2(b) and the relation for the Doppler shift $\mathrm{k}_{\perp}=\mathrm{k}_{\mathrm{x}}=2 \mathrm{k}_{0} \sin \alpha$ is preserved correspondingly. It is important to note that similarly shaped BQOs were observed in experiments on the Globus-M tokamak (see Figure 2c) $[3,4]$. The similarity of BQFs in the linear and nonlinear case, on the one hand, explains the fact that similar BQFs were observed 
on the both tokamaks Globus-M and ASDEX-Upgrade [3-6], in which a significant difference in filament amplitudes was expected. On the other hand, since IQ signals were recorded in relative units in the experiment, it was not possible to determine which scattering regime was observed.
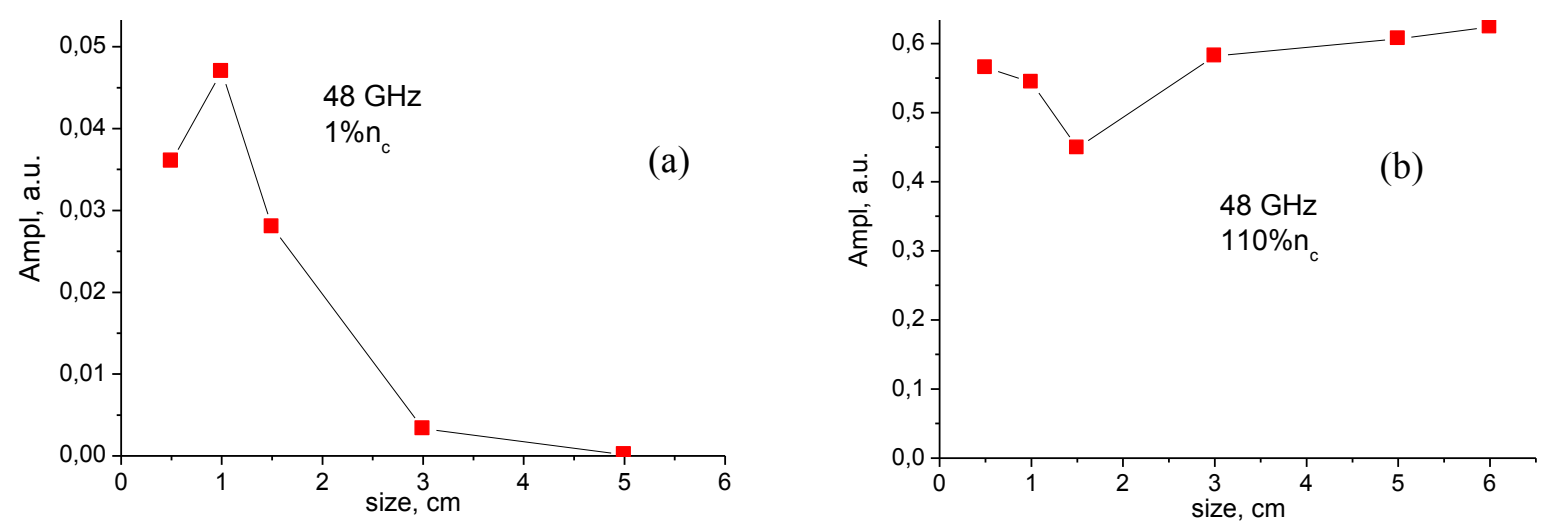

Figure 3. Signal amplitude on filament size dependence in linear (a) and non-linear (b) case.

The next step in the simulation was to determine the dependence of IQ signals on the size of the filament. For the size of $\pi / 2 \mathrm{k}_{\mathrm{i}} \sin \alpha$ in the linear mode, the maximum backscattering intensity should be observed. If the size of the filament exceeds the $\pi / 2 \mathrm{k}_{\mathrm{i}} \sin \alpha$ value, it is expected that the efficiency of backscattering in the Born approximation will decrease due to the process of averaging over the alternating weight function, as it is shown in Figure 3a. On the contrary the signal amplitude calculated using full wave code in non-linear case is slightly depend on filament size as it is seen from figure 3(b).

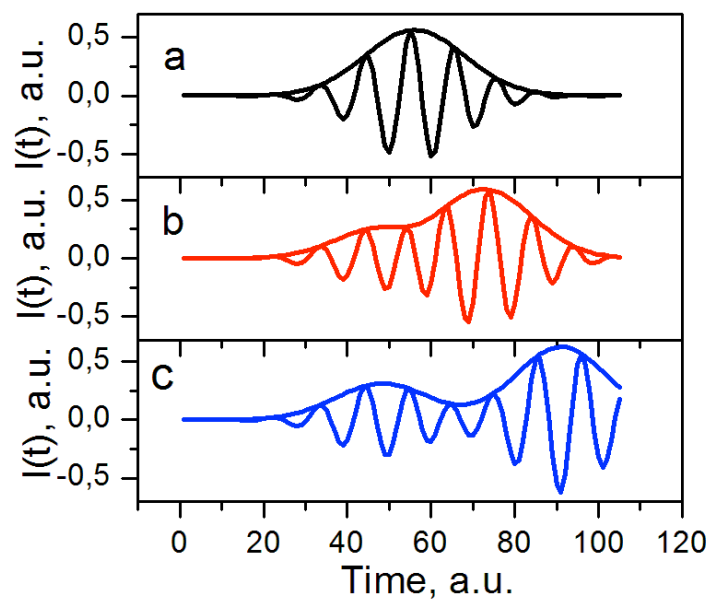

Figure 4. Calculated I(t) signals and its envelops for filament amplitude $-100 \%$, probing frequency $48 \mathrm{GHz}, \mathrm{a}-$ filament size $0.5 \mathrm{~cm}, \mathrm{~b}-3 \mathrm{~cm}, \mathrm{c}-6 \mathrm{~cm}$.
Figure 4 shows how BQFs and their envelopes are deformed in the case of strongly nonlinear scattering. BQFs, similar to those that arise in the linear case, are observed in the calculations in the nonlinear mode only for a small filament size < $\pi / 2 \mathrm{k}_{\mathrm{i}} \sin \alpha$. As the filament size increases, BQF lengthens, while a second maximum appears in the BQF envelope (see Figure $4 d$ and 4c). Moreover, only in the time interval of 30-60 rel. units Doppler frequency shift satisfies the relation $\Delta \omega_{\mathrm{D}}=\mathrm{k}_{\mathrm{x}} \mathrm{V}$. 

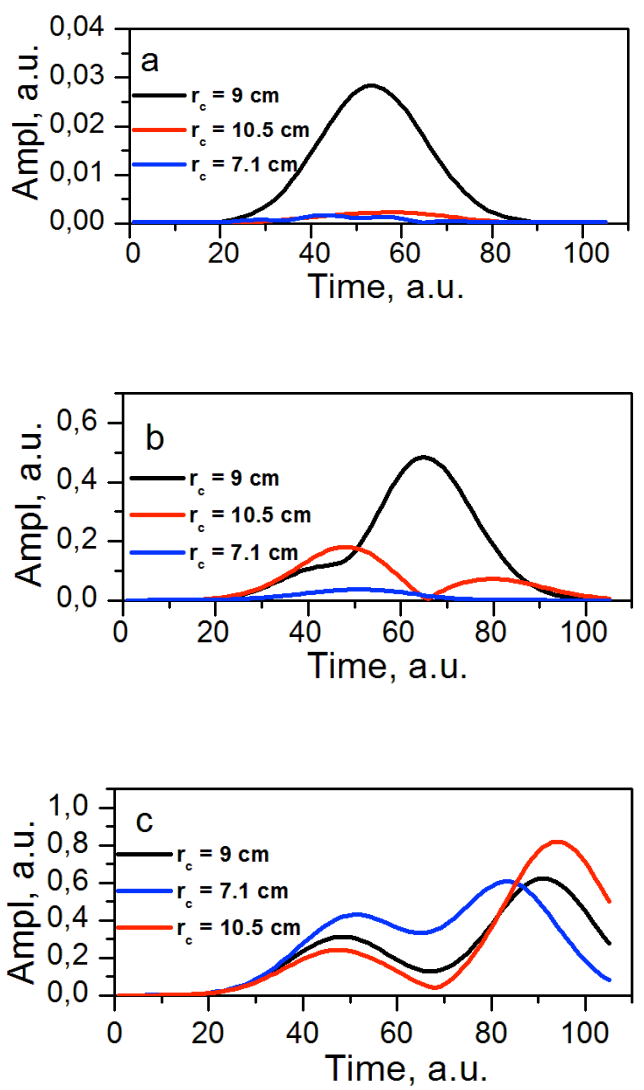

Figure 5. Amplitude of IQ-signals for a - filament amplitude $1 \%$, filament size $1,5 \mathrm{~cm}, \mathrm{~b}-70 \%, 1.5 \mathrm{~cm}, \mathrm{c}$ $-100 \%, 6 \mathrm{~cm}$.

The calculations for the case of multiple frequency probing were carried out for the same radial position of the moving filament $\mathrm{r}=9 \mathrm{~cm}$. Thus, the dependences of IQ signals on the distance between the filament path and cutoffs were obtained, which proves the radial resolution of the method. The signals were calculated in the linear case for a filament size of $1.5 \mathrm{~cm}$ (Figure 5a). In the nonlinear case, this was done for sizes of 1.5 and $6 \mathrm{~cm}$. (Figure $5 \mathrm{~b}$ and $\mathrm{c}$ ). It can be seen that in the linear case, the displacement of the filament trajectory relative to the cutoff by a distance of 1.5-2 $\mathrm{cm}$ leads to the drastic decrease in the signal amplitude by more than an order of magnitude, which indicates a good radial resolution of the method. In the nonlinear mode, for a filament size of the $\pi / 2$ kisin $\alpha$, the radial resolution is noticeably weaker (see Figure 5b). Finally, in a strongly nonlinear mode, when a large size filament moves in the poloidal direction, it is not possible to determine the radial position of its trajectory. The envelopes of signals with two maxima appear again in the calculations. It could be declared that such BQFs with two maxima are a hallmark of a strongly nonlinear backscattering from a large size filament. The BQFs signals similar to those shown in Figure $4 \mathrm{c}$ were not observed in the Globus-M experiments, however, this does not mean the absence of nonlinear scattering in these experiments, in which, as noted, BQFs with one maximum can be observed (see Figure 4a).

\section{Discussion and conclusion}

Full-wave modeling revealed a number of particularities in the transition from linear to nonlinear backscattering from filaments. Firstly, the modeling has shown that similar BQFs are formed in case of scattering off from filament either in case of linear or non-linear regimes, if the filament size does not exceed significantly the characteristic value of $\pi / 2 \mathrm{k}_{\mathrm{i}} \sin \alpha$. In this case, the Doppler shift is determined by the expression, which is also valid in the Born 
approximation in slab geometry. Secondly, with an increase in the amplitude and size of the filament, $\mathrm{BQF}$ deforms forming the double-maximum $\mathrm{BQF}$.

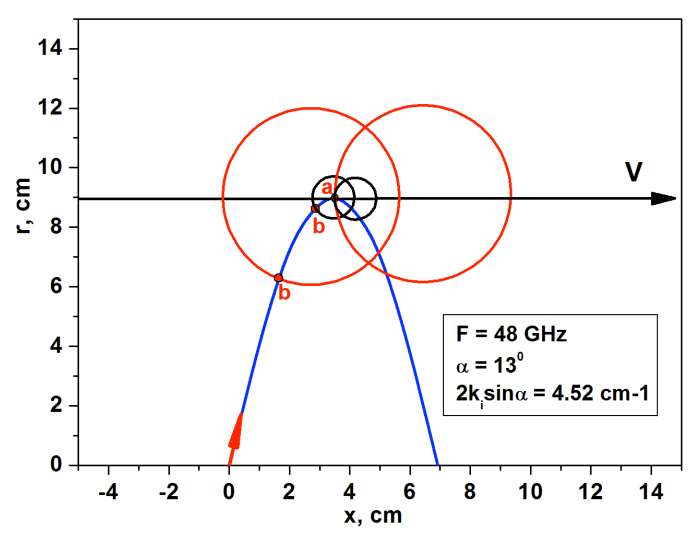

Figure 6. Schematic representation of back reflection from filaments of different sections
The both results of full-wave simulation can be interpreted basing on the view that at a large filament amplitude, when the local plasma density in the filament becomes higher than critical, a transition from backscattering to reflection occurs. The reflection process is schematically shown in Figure 6, where an incident beam raytracing and a circle of critical local plasma density in filaments of different sizes are presented.

It can be seen that when the filament is located to the right of the "a" point, the back reflection occurs in the cutoff and the Doppler shift, as in the case of linear backscattering, is equal to $\Delta \omega_{\mathrm{D}}=\mathrm{k}_{\perp} \mathrm{V},\left(\mathrm{k}_{\perp}=\mathrm{k}_{\mathrm{x}}=2 \mathrm{k}_{0} \sin \alpha\right)$. Back reflection is also possible with a displacement of filaments at points " $b$ ", However, the Doppler shift will change: $\Delta \omega_{D}=k_{\perp}(b) V \cos \beta$, where $\mathrm{k}_{\perp}(\mathrm{b})$ is the wave number of the probing radiation, $\beta$ is the angle between the velocity vector and the wave vector $\mathbf{k}_{\perp}(\mathrm{b})$. This shift at a small filament size slightly differs from the value for the linear case, so that the single maximum BQF shown in Figure 4a are generated. With a large filament size, the appearance of reflections from points "a" and "b" is noticeably spaced in time, resulting in signals similar to those shown in Figure 4c. It is clear that backward reflection also occurs at intermediate points, since at the final aperture of the antenna there are partial incident waves with different ray paths. All of these backscattering and reflection processes are taken into account when calculation using the full-wave code.

The approach proposed in this paper allows us as well to hold calculations of modeling backscattering and reflection processes from filaments with various cross-sectional shapes moving along different paths using the full-wave code IPF-FD3D. The purpose of this simulation is to describe the variety of BQF performances that are observed in the experiment. However, it should be borne in mind that the presented modeling has a number of disadvantages. The calculations have been performed in O-mode using simple slab model and have not taken into account complicated magnetic field configuration taking place in real experiment. Such a task that is difficult even in the optical approximation particularly for tokamaks with a low aspect ratio. Moreover, in presented computation turbulence effects are not taken into account as well. 
Acknowledgements. The work is supported by RSCF grant 18-72-10028 whereas the maintenance of Globus-M tokamak and standard discharge diagnostics systems was supported by the Ioffe Institute.

\section{References}

[1] M. Spolaore et al 2017 Nucl. Mater. Energy 12844

[2] P.B. Snyder et al 2009 Nucl. Fusion 49085035

[3] V.V. Bulanin et al 2011 Tech. Phys. Lett. 37340

[4] V.V. Bulanin et al 2019 Nucl. Fusion 59096026

[5] P. Hennequin et al 44th EPS Conference on Plasma Physics, Belfast, 2017, P1.167

[6] E. Trier et al 45th EPS Conference on Plasma Physics, Prague, 2018, P1.1023

[7] M Hirsch e al 2001 Plasma Phys. Control. Fusion 43 1641-1660

[8] E. Blanco and T. Estrada 2008 Plasma Phys. Control. Fusion 50095011

[9] Lechte C et al 2017 Plasma Phys. Contr. Fusion, 59075006

[10] Bulanin V.V, Yafanov M.V. 2006 Plasma Phys. Rep. 3247 